\title{
Corrigendum: A Curiosity-Based Learning Method for Spiking Neural Networks
}

\author{
Mengting Shi ${ }^{1,2 \dagger}$, Tielin Zhang ${ }^{1 \dagger}$ and Yi Zeng ${ }^{1,2,3,4 * t}$ \\ ${ }^{1}$ Research Center for Brain-inspired Intelligence, Institute of Automation, Chinese Academy of Sciences, Beijing, China, \\ ${ }^{2}$ University of Chinese Academy of Sciences, Beijing, China, ${ }^{3}$ Center for Excellence in Brain Science and Intelligence \\ Technology, Chinese Academy of Sciences, Shanghai, China, ${ }^{4}$ National Laboratory of Pattern Recognition, Institute of \\ Automation, Chinese Academy of Sciences, Beijing, China
}

Keywords: curiosity, spiking neural network, novelty, STDP, voltage-driven plasticity-centric SNN

\section{A Corrigendum on}

A Curiosity-Based Learning Method for Spiking Neural Networks by Shi, M., Zhang, T., and Zeng, Y. (2020). Front. Comput. Neurosci. 14:7. doi: 10.3389/fncom.2020.00007

OPEN ACCESS

Edited and reviewed by:

Huajin Tang,

Zhejiang University, China

*Correspondence:

Yi Zeng

yi.zeng@ia.ac.cn

†These authors have contributed equally to this work

Received: 21 February 2020

Accepted: 23 March 2020

Published: 15 April 2020

Citation:

Shi M, Zhang T and Zeng Y (2020)

Corrigendum: A Curiosity-Based Learning Method for Spiking Neural Networks.

Front. Comput. Neurosci. 14:28. doi: $10.3389 /$ fncom.2020.00028
In the original article, there was an error. In the original main text, there was an inaccurate statement sentence the result of NETalk in Table 3.

A correction has been made to Experiments, The validation of CBSNN on other datasets:

- NETtalk (Sejnowski and Rosenberg, 1987) is usually used for speech generation, consisting 5,033 training and 500 test. The input is a string of letters with fixed length of 7 , which is encoded into 189 dimensions (each character has a 27 length one-hot vector). The output is 26 dimensions which represent 72 phonetic principles. For this mapping task with strong global regularities, VPSNN reaches 0.8680 accuracy. Although CBSNN is only slightly higher than VPSNN, it saves about half of the computation cost.

The authors apologize for this error and state that this does not change the scientific conclusions of the article in any way. The original article has been updated.

\section{REFERENCES}

Sejnowski, T. J., and Rosenberg, C. R. (1987). Parallel networks that learn to pronounce english text. Compl. Syst. 1, 145-168.

Copyright (c) 2020 Shi, Zhang and Zeng. This is an open-access article distributed under the terms of the Creative Commons Attribution License (CC BY). The use, distribution or reproduction in other forums is permitted, provided the original author(s) and the copyright owner(s) are credited and that the original publication in this journal is cited, in accordance with accepted academic practice. No use, distribution or reproduction is permitted which does not comply with these terms. 